\title{
El tratamiento de deprivación androgénica en pacientes con cáncer de próstata no se asocia a un aumento de riesgo cardiovascular
}

Androgen deprivation therapy in patients with prostate cancer is not associated with an increase in cardiovascular risk

Nguyen P. y col JAMA 2011;306:2359-2366

\section{Objetivos}

Sintetizar la evidencia científica respecto de la asociación existente entre los tratamientos de deprivación androgénica y la mortalidad de causa cardiovascular (CV) y específica por cáncer en pacientes con cáncer de próstata no metastásico de riesgo desfavorable (CPNMRD)

\section{Fuentes de datos}

Publicaciones en inglés en MEDLINE y EMBASE desde 1996 a 2011, y en el Registro Central Cochrane de ensayos clínicos hasta 2011.

\section{Selección de estudios}

Estudios aleatorizados controlados con más de un año de seguimiento, que hubieran incluido pacientes con CPNMRD sensible a hormonas, en tratamiento con análogos LHRH y que hubieran registrado información sobre mortalidad CV.

\section{Extracción de datos}

Los datos fueron extraídos por dos revisores independientes. Fue considerada relevante la información sobre el tipo y la duración de la deprivación androgénica, sobre el número de muertes (totales, CV y por cáncer prostático), y sobre el tiempo medio de seguimiento.

\section{Resultados principales}

Se detallan en la tabla 1.

Tabla1: riesgo relativo de mortalidad global, cardiovascular y por cáncer de próstata, entre las dos ramas de tratamiento comparadas. Los tiempos medios de seguimiento de los diferentes trabajos oscilaron entre 6,8 y 11 años.

\begin{tabular}{|c|c|c|c|c|}
\hline \multicolumn{3}{|c|}{$\begin{array}{l}\text { Eventos sobre el total de participantes } \\
\text { (eventos/n) y mortalidad por grupo }\end{array}$} & \multirow{2}{*}{$\begin{array}{l}\text { Riesgo } \\
\text { relativo } \\
\text { (IC95\%) }\end{array}$} & \multirow[b]{2}{*}{ Heterogeneidad } \\
\hline & $\begin{array}{l}\text { Deprivación } \\
\text { androgénica }\end{array}$ & Control & & \\
\hline Global & $\begin{array}{c}1140 / 2527 \\
(37,7 \%)\end{array}$ & $\begin{array}{c}1213 / 2278 \\
(44,4 \%)\end{array}$ & $\begin{array}{c}0,86 \\
(0,80 \mathrm{a} 0,93)\end{array}$ & $\begin{array}{c}Q=16,86 ; p=0,08 ; \\
I^{2}=40,7 \%\end{array}$ \\
\hline Cardiovascular & $\begin{array}{c}255 / 2200 \\
(11 \%)\end{array}$ & $\begin{array}{c}252 / 1941 \\
(11,2 \%) \\
\end{array}$ & $\begin{array}{c}0,93 \\
(0,79 \text { a } 1,10) \\
\end{array}$ & $\begin{array}{c}Q=5,12 ; p=0,64 ; \\
\left.\right|^{2}=0 \%\end{array}$ \\
\hline $\begin{array}{l}\text { Específica por } \\
\text { cáncer de próstata }\end{array}$ & $\begin{array}{c}443 / 2527 \\
(13,5 \%)\end{array}$ & $\begin{array}{c}552 / 2278 \\
(22,1 \%)\end{array}$ & $\begin{array}{c}0,69 \\
(0,56 \mathrm{a} 0,84)\end{array}$ & $\begin{array}{c}Q=24,57 ; p=0,006 ; \\
I^{2}=59,3 \%\end{array}$ \\
\hline
\end{tabular}

\section{Conclusiones}

En pacientes con CPNMRD no pudo documentarse asociación entre el uso de terapia de deprivación androgénica y la mortalidad CV, asociándose su uso a una mejora en la sobrevida global y específica por cáncer de próstata.

\section{Comentario}

En los últimos años y sobre información proveniente de estudios retrospectivos, se ha correlacionado la utilización de bloqueo androgénico (generalmente con análogos LHRH) con la aparición de trastornos metabólicos (diabetes hipercolesterolemia) y CV (infarto agudo de miocardio y muerte súbita cardiaca), lo que condujo a numerosas discusiones en congresos y a la publicación de artículos alertando sobre los riesgos de este tipo de tratamiento.

Los autores del artículo que hemos resumido realizaron un meta-análisis sobre publicaciones prospectivas aleatorizadas que habían comparado la utilización de análogos LHRH contra un grupo control y documentaron que dicho bloqueo no se asocia a un aumento del riesgo $\mathrm{CV}$, incluso en pacientes con más de tres años de tratamiento. Tampoco pudieron detectar diferencias de resultado en los diferentes subgrupos de estrategias de bloqueo androgénico que pudieron ser evaluadas.

Vale destacar que en el subgrupo de pacientes con insuficiencia cardiaca congestiva e infarto de miocardio se observó una tendencia hacia una mayor incidencia de eventos CV en el subgrupo asignado a terapia de deprivación androgénica, que pudo no haber cobrado significancia estadística por falta de poder para detectar este tipo de diferencias en subgrupos. Por otro lado, los estudios seleccionados para este meta-análisis fueron de fase 3 , lo que implica una selección de paciente más sanos que la población que solemos tratar en nuestra práctica clínica habitual y que podría ser más vulnerable a eventos CV y no ajustarse estrictamente a las conclusiones de este metaanálisis; y que en Argentina suele usarse también la ciproterona en altas dosis diarias (300 mg), opción que no fue evaluada en los artículos incluidos en esta revisión sistemática'.

\section{Conclusiones del comentador}

Considero que este meta-análisis aporta una renovada visión respecto de la seguridad $\mathrm{CV}$ de los tratamientos de bloqueo androgénico, quedando todavía dudas si realmente la población de la que fueron sacadas sus conclusiones es representativa de la población que solemos atender en nuestra práctica habitual.

Pablo Martínez [ Servicio de Urología del Hospital Italiano de Buenos Aires, pablo.martinez@hospitalitaliano.org.ar ]

Martínez P. El tratamiento de deprivación androgénica en pacientes con cáncer de próstata no se asocia a un aumento del riesgo cardiovascular. Evid Act Pract Ambul. Ene-Mar. 15(1). 20. Comentado de: Nguyen P y col. Association of Androgen Deprivation Therapy With Cardiovascular Death in Patient With Prostate Cancer. A Meta-analysis of Randomized Trials. JAMA 2011; 306:2359-2366.

Referencias

1. Schröder F. Antiandrogens as monotherapy for prostate cancer. Eur Urol 1998;38 Suppl 3:12-17. 